\title{
New Analytical Methods Developed for Determination of Perfluorinated Surfactants in Waters and Wastes ${ }^{\dagger}$
}

\author{
Marek Trojanowicz, ${ }^{*}$ Lena Wójcik, Jacek Musijowski, Mariusz Koc, Ewa Poboży, and Edyta Król \\ Department of Chemistry, University of Warsaw, Pasteura 1, 02-093 Warsaw, Poland
}

RECEIVED OCTOBER 13, 2010; REVISED MAY 12, 2011; ACCEPTED MAY 13, 2011

\begin{abstract}
Perfluorinated organic compounds have been recognized in recent years as globally distributed persistent organic pollutants of an entirely anthropogenic origin, but present already even in the most remote places of the globe, including organisms of wild fauna. Numerous studies have also shown that they are contained in human organisms all over the world. In this work a special attention is given to perfluorinated carboxylic acids (PFCAs), widely used in various areas of modern life. New methods developed for their determination using the HPLC with fluorescence detection and capillary electrophoretic methods are discussed, as well as the new method for the determination of total organic fluorine (TOF). (doi: 10.5562/cca1776)
\end{abstract}

Keywords: perfluorinated organic compounds, surfactants, HPLC, capillary electrophoresis, total organic fluorine, water analysis

\section{INTRODUCTION}

A widespread presence and environmental persistence of the perfluorinated organic compounds (POC) received a worldwide attention in recent two decades. ${ }^{1}$ In recent decades they have been widely produced for various applications as stable and efficient surfactants, being utilized in syntheses of fluorinated polymers, and are applied in household products and cosmetics. Their unusual stability in the environment and their resistance to chemical and biochemical degradation result in a wide global proliferation, including remote regions without any anthropogenic activity, ${ }^{2}$ therefore many of POCs are considered as persistent organic pollutants (POPs). ${ }^{3}$ They are commonly present in human and animal organisms. Their accumulation in particular organs and incorporation into a lipid cell walls ${ }^{4}$ are commonly known. The two most commonly detected types of perfluorooctanoic acid (PFOA, C8 PFCA) and perfluorooctane sulfonic acid (PFOS) are globally present in human blood and serum samples at ppb $\left(\mu \mathrm{g} \mathrm{L}^{-1}\right)$ level. Some of their essential positive features should also be mentioned. In the automotive field alone the use of a fluoropolymer layer on fuel hoses reduces the fuel permeation by $95 \%$ and reduces the volatile emissions and improves the fuel economy. Aqueous film-forming foams (AFFF) have saved lives and reduced property damage in airports and on deep-water oil rigs.
Monitoring of their concentration in trace level in complex matrices is a serious analytical challenge. It requires usually a complex sample clean-up and preconcentration of trace analytes. Because of a large variety of perfluorinated compounds present in environmental samples the best results can be obtained using the highperformance chromatography with mass spectrometry detection (LC/MS). ${ }^{5}$ For this purpose also some other detection methods can also be employed such as the fluorescence $^{6,7}$ with appropriate derivatization of analytes or the conductometric ${ }^{8}$ one. For the purpose of the environmental monitoring of total amount of fluorinated organic types (total organic fluorine, TOF') several methods of their decomposition by combustion ${ }^{10}$ or chemical reduction ${ }^{11-13}$ have been developed and combined with different methods of fluoride determination. The methods of environmental analysis, developed in our research group, are described below in detail.

\section{EXPERIMENTAL}

\section{Analytical Methods for Determination of PFCAs}

Determination of POCs is an analytical challenge. Reliable methods of extraction, separation and identification in complex matrixes are necessary. The difficulty of the determination of the most commonly occurring perfluorinated carboxylic acids (PFCAs) is their very

\footnotetext{
$\uparrow$ Presented at the $10^{\text {th }}$ International Symposium and Summer School on Bioanalysis within the CEEPUS Network CII-HU-001004-0910, Zagreb, Croatia, July 2010.

* Author to whom correspondence should be addressed. (E-mail: trojan@chem.uw.edu.pl)
} 

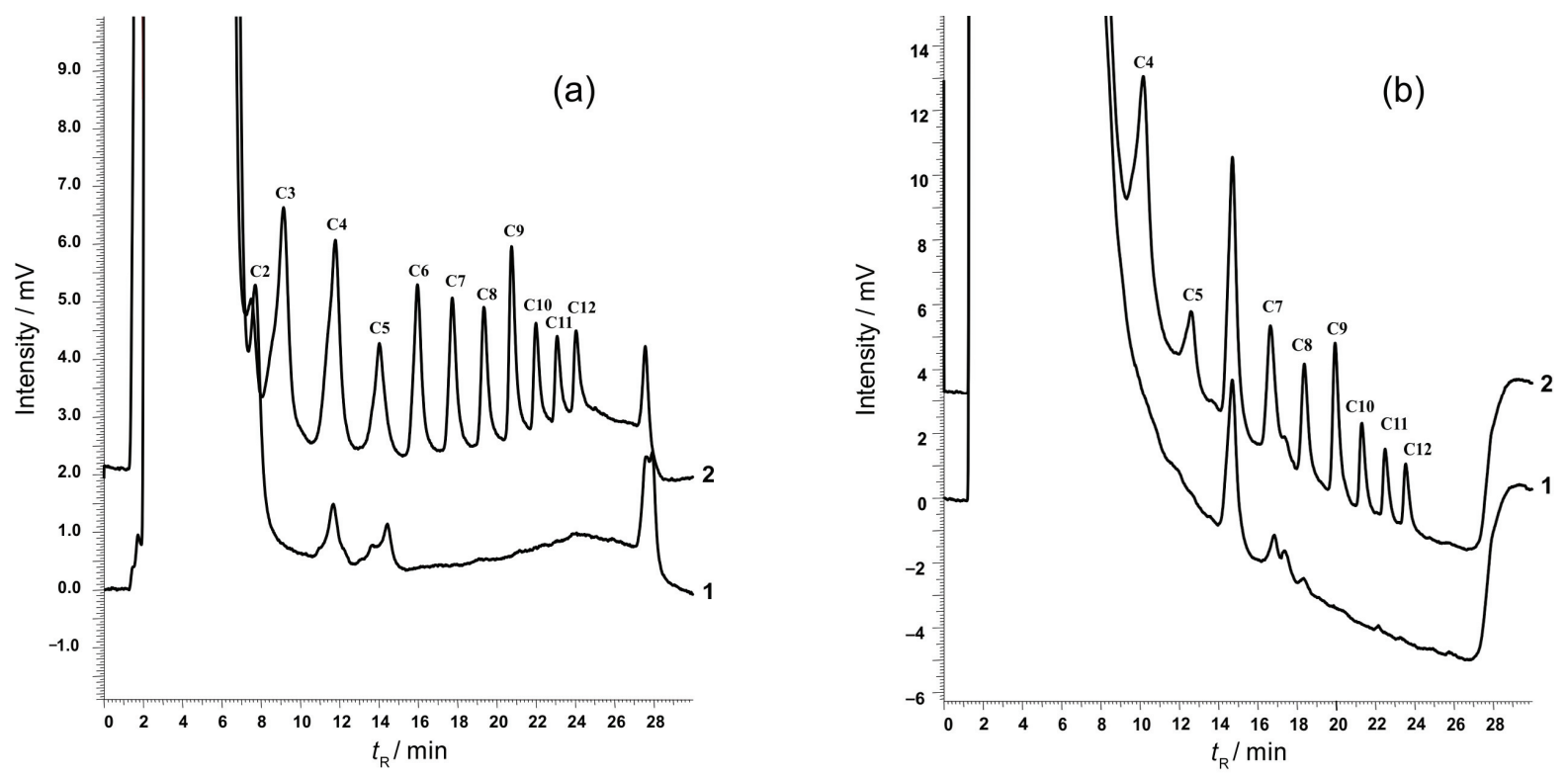

Figure 1. Chromatograms obtained for blanks (1) and mixtures of $0.5 \mathrm{mg} \mathrm{L}^{-1}$ PFCA standards (2) for derivatization using solution of 3-BrAC, $\gamma(3-\mathrm{BrAC})=2 \mathrm{~g} \mathrm{~L}^{-1}$ (Figure 1a) and solution of BrMMC, $\gamma(\mathrm{BrMMC})=2 \mathrm{~g} \mathrm{~L}^{-1}$ (Figure 1b). Detection at $306 / 420 \mathrm{~nm}$ for $3-\mathrm{BrAC}$ and $330 / 390 \mathrm{~nm}$ for BrMMC. ${ }^{7}$

low concentration in the samples (ng-pg $\mathrm{L}^{-1}$ or ng-pg $\mathrm{g}^{-1}$ ) and their weak absorption of the UV radiation. Numerous analytical methods have been developed to determine PFCAs and most of them are chromatographic. ${ }^{14,15}$ For determination of PFCAs without derivatization, the LC MS/MS, ${ }^{5,16,17}$ the LC-TOF ${ }^{18}$ or with derivatization the GC MS ${ }^{19}$ and the GC ECD ${ }^{20}$ are commonly used in the environmental and biological samples. The ion chromatography with conductimetric detection for the separation PFCAs having C3 -8 has been also proposed ${ }^{8}$ with the LODs in range $0.12-0.66 \mathrm{mg} \mathrm{L}^{-1}$, while with the SPE determination of $50 \mu \mathrm{g} \mathrm{L}^{-1}$ was possible.

Different approaches using solid-phase extraction methodology for preconcentration of PFCAs have been reported. For determination with the LC-MS, the methodology of Moody et al. ${ }^{21}$ employing C18 sorbents limited to long chain acids has been employed by various authors. There has recently also been developed the application of polymeric sorbents from Waters Oasis HLB and Oasis WAX mixed-mode weak anion-exchange reversed phase for PFCAs preconcentration. ${ }^{22}$

\section{HPLC Method With Fluorescence Detection}

In order to apply the fluorimetric detection of PFCAs, Ohya et al. ${ }^{6}$ reported the derivatization with laboratory synthesized 3-bromoacetyl-7-methoxycoumarin (BrAMC), while conditions of carrying out reaction and separation were optimized. With the isocratic elution, all derivatized analytes were separated during $18 \mathrm{~min}$. The developed method was applied for the determination of PFCAs in rats liver and recoveries $>90 \%$ were obtained for $50 \mathrm{mg}$ homogenate spiked with $1 \mathrm{nmol} \mathrm{L}{ }^{-1}$ PFCAs.
The aim of our work was to develop a HPLC method for determination of PFCAs with fluorimetric detection based on the use of commercially available fluorophores. ${ }^{7}$ The studies were focused on the investigation of two coumarin fluorophores similar to 3-bromoacetyl-7-methoxycoumarin mentioned above. Both fluorophores employed in this work, namely 3-bromoacetylcoumarin (3-BrAC) and 4-bromomethyl7-methoxycoumarin (BrMMC), are commercially available, and the latter one has already been widely employed for derivatization of carboxylic acids for the HPLC determination with fluorimetric detection of e.g. fatty acids. ${ }^{23}$

Among the reagents used in derivatization for application of fluorescence detection in HPLC, coumarin derivatives present one of the most sensitive compounds. The yield of derivatization with coumarin derivatives, limit of detection and efficiency of separation of derivatized analytes depend on numerous experimental factors. Using a standard mixture containing PFCAs from 2 to 12 carbon atoms (C2 to $\mathrm{C} 12)$ at concentrations of $0.5 \mathrm{mg} \mathrm{L}^{-1}$ each, for both derivatives effect of reagent concentration used for derivatization, reaction time, temperature of reaction and kind of solvent were examined.

Comparison of two reagents used for derivatization was made in $70{ }^{\circ} \mathrm{C}$, for a reaction time of $60 \mathrm{~min}$ and concentration of reagents of $2 \mathrm{~g} \mathrm{~L}^{-1}$. The examples of obtained chromatograms with corresponding recordings for blanks are shown in Figure 1. In a similar period of time of about 25 min the separation of all analytes and better base-line were obtained for 3-BrAC. 
Surface areas for recorded peaks with two coumarin derivatives are very similar. A much larger signal for excess of reagent recorded for BrMMC makes impossible recording signals for $\mathrm{C} 2$ and $\mathrm{C} 3$. A much larger signal recorded for $\mathrm{C} 6$ with BrMMC is caused by unidentified solutes present in the blank. For further investigations 3-BrAC was selected as derivatizing reagent.

Effect of the temperature on derivatization reaction was examined in the range from 20 to $70{ }^{\circ} \mathrm{C}$ for reaction time of $60 \mathrm{~min}$. At the highest examined temperature of derivatization of $70{ }^{\circ} \mathrm{C}$ also effect of derivatization time was investigated in the range from 20 to $120 \mathrm{~min}$. The role of this parameter is smaller, and decrease of signal magnitude at 120 min for most analytes was attributed to the loss of analytes during long time derivatization. As some loss of more volatile species can be expected also at the highest examined temperature, as the most safe and efficient conditions of derivatization were selected $60 \mathrm{~min}$ derivatization at $70{ }^{\circ} \mathrm{C}$. Due to the large differences in affinity of the examined analytes to $\mathrm{C} 18$ stationary phase in the used reversedphase system, their efficient separation in a reasonable time period can only be obtained with gradient elution.

In the optimized conditions of derivatization the determinations of PFCAs were carried out in a concentration range from 0.025 to $0.5 \mathrm{mg} \mathrm{L}^{-1}$ in injected solutions. For a $50 \mu \mathrm{L}$ sample volume satisfactory linearity of the response was obtained and limits of detection (for $\mathrm{S} / \mathrm{N}=3$ ) in the range from 5 to $10 \mu \mathrm{g} \mathrm{L}^{-1}$. In studies of repeatability of derivatization procedure with $3-\mathrm{BrAC}$ in optimized conditions, for 5 repetitions carried out during 5 consecutive days, RSD values for peak areas for all the analytes were below $1 \%$. No significant differences in the efficiency of separation were also observed. The derivatized analytes were stable in time, as after two days of storage recorded chromatograms were practically the same as those recorded immediately after derivatization. No additional by-products after 2 days of storage were observed.

The detection limits obtained in the developed procedure are too high for a direct determination of PFCAs at level present in natural waters ${ }^{21,24}$ so the next step in development of a complete procedure was the optimization of a solid-phase extraction method for the preconcentration of analytes from diluted natural samples. Based on a vast literature on the application of different sorbents for this purpose, ${ }^{25}$ two commercially available sorbents were selected, namely Sep-PAK C18 and Oasis-WAX. Besides the optimization of elution mode for retained species on sorbent bed, another essential factor was to eliminate traces of water from the extracts, which interferes in the derivatization reaction.

Because of the strong retention of examined analytes on $\mathrm{C} 18$ sorbent bed, recovery of the elution was investigated using three $2.5 \mathrm{~mL}$ fractions of methanol.
The obtained data for 100 -fold preconcentration using $500 \mathrm{mg}$ sorbent bed showed a satisfactory recovery (above $80 \%$ ) with single portion of only methanol for C4, C7 and C9 PFCAs. The total recovery obtained by means of three fractions of eluting methanol was more then $80 \%$ for all examined analytes from $\mathrm{C} 3$ to $\mathrm{C} 12$, except C2 where much lower total recovery was found. Reproducibility of this procedure was also satisfactory, as RSD was obtained for various analytes in the range from 0.2 to $1.4 \%$. For 1000 -fold preconcentration from a $1 \mathrm{~L}$ sample containing $0.3 \mu \mathrm{g} \mathrm{\textrm {L } ^ { - 1 }}$ of each PFCA, a satisfactory total recovery above $90 \%$ was obtained for C4 to C6 PFCAs, only, whereas for other examined analytes it was much lower, and non-acceptable for practical applications.

Similar studies were carried out for Oasis-WAX cartridges, additionally investigating the role of an amount of the sorbent taken for preconcentration. As according to the manufacturer this sorbent can be used for partly polar analytes with $\mathrm{p} K_{\mathrm{a}}$ values about or more than $1.0\left(\mathrm{p} K_{\mathrm{a}}\right.$ for PFOA is 2.5$)$ so, it seems to be suitable for the examined analytes with their retaining based on both hydrophobic and ion-exchange interactions. For the elution of retained species methanol containing ammonia is recommended. Because of this, in the first step the effect of two levels of ammonia 0.1 and $5 \%$ respectively in methanol was investigated for 100preconcentration using a $60 \mathrm{mg}$ sorbent bed. A better recovery was observed for a smaller concentration of ammonia in methanol, hence such conditions were used in further experiments. One can also see, however, that in these conditions a satisfactory elution was observed

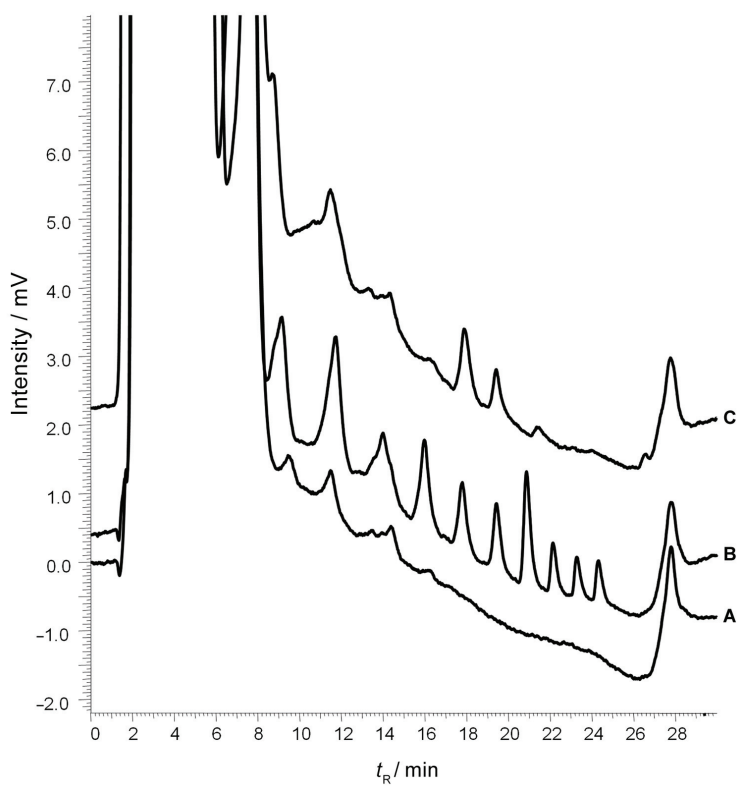

Figure 2. Chromatograms obtained for natural water sample after 100-fold preconcentration: blank (A); standard solution, $c=0.1$ $\mathrm{mg} \mathrm{L}^{-1}$ (without preconcentration) (B); water sample (C). ${ }^{7}$ 
for few PFCAs, only, namely C4, C5, C6 and C9. Alteration of $\mathrm{pH}$ value of preconcentrated solutions in the range of 5.0 to 8.0 did not affect the observed recovery. Based on the obtained experimental data, C18 sorbent Sep-PAK was considered as more suitable for preconcentration of trace amounts of PFCA from environmental samples.

A complete procedure with 100 -fold preconcentration with C18 Sep-PAK $500 \mathrm{mg}$ cartridge was employed for determination of PFCAs in surface water sample collected in an industrial region close to Tarnów, a city in the Southern Poland, where a large chemical plant producing fluorinated compounds is based. The chromatograms recorded for blank, standard mixture of PFCAs, and analyzed sample are shown in Figure 2. Based on retention times, two PFCA analytes were identified, namely $\mathrm{C} 7$ and $\mathrm{C} 8$ (PFOA) at concentrations 1.0, 0.63 $\mu \mathrm{g} \mathrm{L}^{-1}$, respectively. Recoveries of $93.2 \%$ and $99.3 \%$ for $\mathrm{C} 7$ and $\mathrm{C} 8$ spike in natural water were obtained.

\section{Capillary Electrophoresis Methods for Determina- tion of PFCAs}

A very high resolution of capillary electrophoretic (CE) methods and availability of commercial instruments with various detectors is a reason for considering the application of CE methods for determination of perfluorinated surfactants. In our studies we have developed a CE methods for determination of PFCAs with 6 to 12 carbon atoms in alkyl chain with a direct UV detection. ${ }^{26}$ Because of the weak absorption of light by the target PFCA analytes, the detection limits without any additional preconcentration step were from 2 to 33 $\mathrm{mg} \mathrm{L}^{-1}$. We have also developed a CE method based on the use of a capillary electrophoretic microfluidic chip with conductivity detection. ${ }^{27}$ This first attempt resulted with a separation of PFCAs from C6 to C10 carbon atoms and detection limit at the level of 0.3 to 6.7

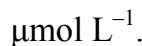

The aim of other studies was to optimize also a capillary electrophoretic method for determination of PFCAs with the indirect spectrophotometric detection. The conducted studies included selection of a chromophore probe and optimization of conditions for the detection and separation of $\mathrm{C} 6$ to $\mathrm{C} 12$ PFCAs.

Strong nucleophilic effect of fluorine atoms in the PFCA molecules causes the perfluorinated acids to be of a moderate strength, hence in alkaline media they are present predominantly as anions. Therefore the optimization of PFCAs separation by the CE to some extent can be based on the existing wide literature on the $\mathrm{CE}$ separation of anions with indirect photometric detection. $^{28}$ The optimization of the background electrolyte (BGE) composition should consider obtaining the best resolution of analytes as well as the best sensitivity of detection in terms of level of detection limits. It generally involves optimization of the content of ionic conducting components in the BGE, organic solvent, chromophore probe, $\mathrm{pH}$ and value of applied voltage.

The optimization of separation of PFCAs from C6 to $\mathrm{C} 12$ was carried out with a single variable procedure, examining successively effect of particular factors. In the preliminary measurements a dinitrobenzoic acid (DNBA) isomer was employed as a chromophore probe because its mobility well matched that of analytes and $40 \%$ methanol content in the BGE. The use of a relatively high content of ionic species in the BGE is necessary to provide sufficient conductivity of the BGE containing a large amount of organic solvent. The effect of Tris content in the BGE was examined in the concentration range from 20 to $100 \mathrm{mmol} \mathrm{L}^{-1}$. No pronounced improvement of resolution was observed with the increase of Tris concentration in the BGE, especially for PFCAs with longer alkyl chain, but it broadened the signal for C6 and C7. As optimum, $50 \mathrm{mmol} \mathrm{L}^{-1}$ Tris concentration in the BGE was chosen.

In the determinations of surfactants which can be strongly adsorbed on the capillary walls, both in the case of anionic species and for cationic ones, measurements have to be carried out with a large content of organic solvent in the BGE. The comparison studies with $40 \%$ of organic solvent in the BGE were carried out for acetone, tetrahydrofurane, methanol and isopropanol. The best peak shape and reproducibility of measurements with the smallest level of baseline noise was observed for methanol, hence methanol was employed in further studies. The $40 \%$ content of methanol in the BGE does not, however, allow to obtain a baseline separation of $\mathrm{C} 9$ to $\mathrm{C} 12$ PFCAs, hence additionally the effect of methanol concentration in the BGE on the migration times and the signal magnitude were examined. The increase of methanol content above $40 \%$ results in longer migration times of PFCAs and enhances the resolution of PFCAs with longer alkyl chains. However, its increase up to $60 \%$ causes evident decrease of the signal magnitude, hence finally $50 \%$ content was chosen as an optimum.

A critical factor to optimize in the CE determination with indirect photometric detection is the selection of a suitable chromophore probe and this has been discussed in the literature. ${ }^{28}$ The 2,4-dinitrobenzoic acid (2,4-DNBA) was employed as a chromophore probe in our experiments. Its spectrum has a maximum of absorption at $\lambda=254 \mathrm{~nm}$, however in the BGE containing a much higher concentration of Tris that also adsorbs at this wavelength, a larger absorption has been observed at $\lambda=270 \mathrm{~nm}$ and this wavelength was used for further studies.

The concentration of chromophore probe affects the migration times of analytes and their limits of detection in the CE determination. In the examined system 
with the BGE containing 2,4-DNBA the changes of chromophore concentration investigated in the range from 2 to $10 \mathrm{mmol} \mathrm{L}^{-1}$ do not affect the migration times significantly. Changes for C12 PFCA were from 17 to $19.5 \mathrm{~min}$, whereas for C7 PFCA from 25.5 to $31 \mathrm{~min}$. This factor then was not deciding in selection of the optimum concentration of the probe. This concentration has, however, significant effect on the signal magnitude and limits of detection of analytes that also is affected by the baseline noise. It was shown in the literature, that the limit of detection (LOD) depends not only on the chromophore concentration, but also on magnitude of transfer ratio and value of ratio of absorption of the BGE to the noise amplitude. The lack of such a direct relationship on the concentration of a chromophore probe has been found in the examined system for the peak height and the area of recorded peaks for analytes. In case of the peak area the lack of monotonous relationship between signal magnitude and concentration of a probe was observed for C6 and C9 PFCAs. Based on the obtained data as optimum concentration of chromophore probes of as $7 \mathrm{mmol} \mathrm{L}^{-1}$ was chosen.

In the optimized conditions the examined analytes C6 to C12 PFCAs are baseline resolved (Figure 3) with a satisfactory stability of the baseline and efficiency of separation which, expressed by a number of theoretical plates was in the range from 150000 to 220000 . Equally good was also the linearity of the calibration curves obtained for the concentration range from 50 to 250 $\mu \mathrm{mol} \mathrm{L}{ }^{-1}$ for all analytes. The reproducibility evaluated for 10 measurements for migration times was in the range from 1 to $2 \%$ RSD (relative standard deviation), while for the peak heights from 2 to $7 \%$ RSD.

The developed method was validated for natural water matrices by fortifying with C6-C12 PFCA three types of waters (river, lake, tap) at two levels. The lower level of the spiking was chosen close to the LOQ of this method and the higher one at the tested linear range of the method $\left(0.025\right.$ and $\left.0.2 \mathrm{mmol} \mathrm{L}^{-1}\right)$. Most recoveries are in the $100 \pm 10 \%$ range and \% RSD is equal or smaller than $5 \%$. This data demonstrates that the method is accurate and precise, well within the range of acceptable recoveries of $70-120 \%$ for environmental analytical methods. Figure 4 presents electropherograms for fortified lake water samples. It is evident that the analytes' signals are not influenced by any interferences. However, the baseline obtained for these natural samples is not as stable as the one obtained for calibration standards. This, however, does not influence the quantification as demonstrated by analyte recoveries.

\section{Determination of Total Organic Fluorine}

Determination of total organic fluorine (TOF) with a sufficient selectivity and a low detection limit is neces-

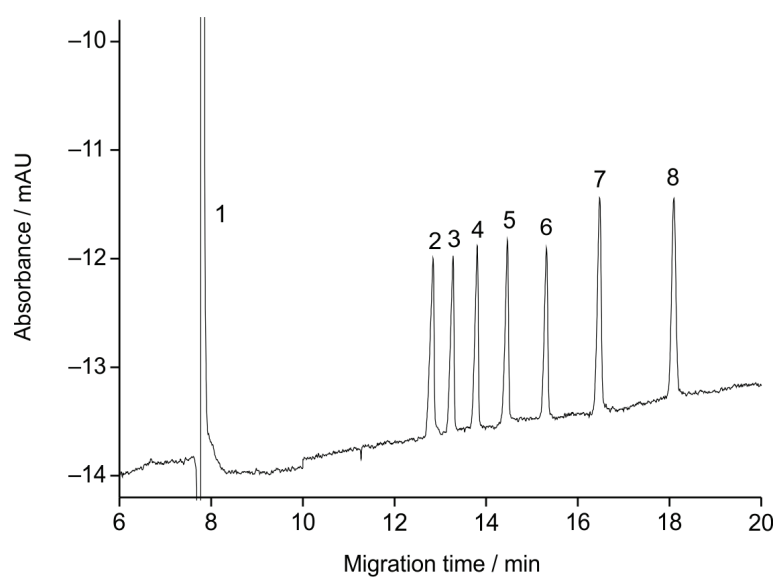

Figure 3. Electropherogram recorded for mixture of PFCAs in optimized conditions: $c$ (PFCA) $=0.1 \mathrm{mmol} \mathrm{L}^{-1}$ each; background electrolyte: Tris $\left(c\right.$ (Tris) $\left.=50 \mathrm{mmol} \mathrm{L}^{-1}\right), 2,4-\mathrm{DNBA}$ $\left(c(2,4-\mathrm{DNBA})=7 \mathrm{mmol} \mathrm{L}^{-1}\right), \mathrm{pH}=9, w($ methanol $)=50 \%$. Applied high voltage $u=+25 \mathrm{kV}$. Hydrostatic injection $3 \mathrm{~s}$ at 0.5 psi, capillary $60 / 50 \mathrm{~cm}$. Signal assignment: $1-5 \mathrm{mmol} \mathrm{L}^{-1}$ DMSO (EOF marker), 2-C12, 3-C11, 4-C10, 5-C9, 6-C8, 7$\mathrm{C} 7$ and 8-C6 PFCA.

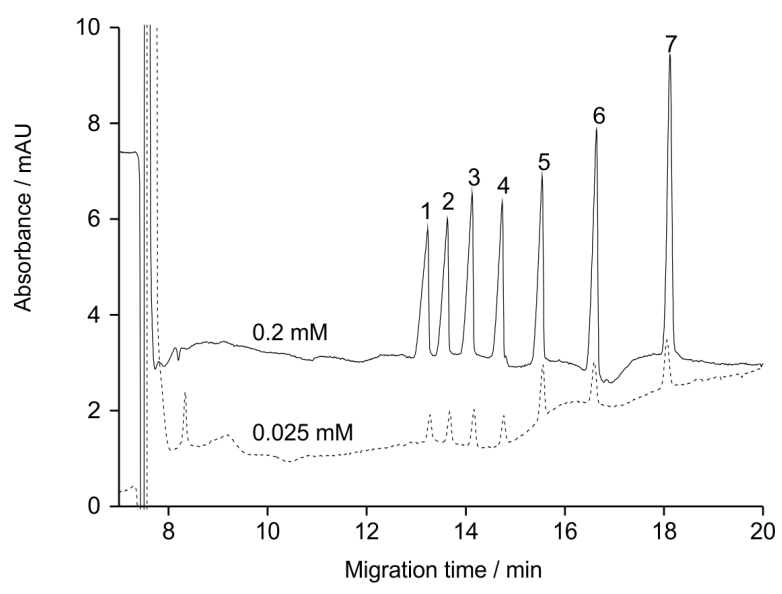

Figure 4. Electropherograms obtained for water lake samples fortified with C6-C12 PFCAs at concentrations of 0.2 and $0.025 \mathrm{mmol} \mathrm{L}^{-1}$.

sary to obtain a mass balance of total organic fluorine in environmental and biological samples, as well as e.g. for monitoring of degradation processes of fluorinated organic compounds. ${ }^{29}$ Such determinations can be carried out directly e.g. by the use of ${ }^{19} \mathrm{~F} \mathrm{NMR},{ }^{21}$ but most commonly they are carried out by release of fluorine from organic compounds and analytical determination of fluoride ion. Release of fluorine can be achieved with different methods, including the combustion with oxygen in the furnace at $900-1000{ }^{\circ} \mathrm{C},{ }^{10}$ or by the reaction with sodium biphenyl, which was employed also in our earlier works on flow-injection methods for TOF determination. ${ }^{11,12}$ 
Table 1. Comparison of different chromatographic methods reported in the literature for determination of PFCAs

\begin{tabular}{lllc}
\hline Method & Sample & LOD & Ref. \\
\hline HPLC-MS /MS & Seawater & $1.8-5.2 \mathrm{pg} \mathrm{L}^{-1}$ (with SPE from 1 L) & 17 \\
HPLC-CD & Industrial process liquids & $50 \mu \mathrm{g} \mathrm{L}^{-1}$ (with SPE from 100 mL) & 8 \\
HPLC-FLD & Liver homogenate & $1 \mathrm{nmol} \mathrm{g}^{-1}$ tissue & 6 \\
HPLC-FLD & Surface water & $43-75 \mathrm{ng} \mathrm{L}^{-1}$ (with SPE from 100 mL) & 7 \\
GC-MS & Harbor seawater & $0.02-0.75 \mu \mathrm{g} \mathrm{L}^{-1}$ (with SPME from 5 mL) & 19 \\
CZE-UV & Standards & $2-33 \mathrm{mg} \mathrm{L}^{-1}$ & 26 \\
\hline
\end{tabular}

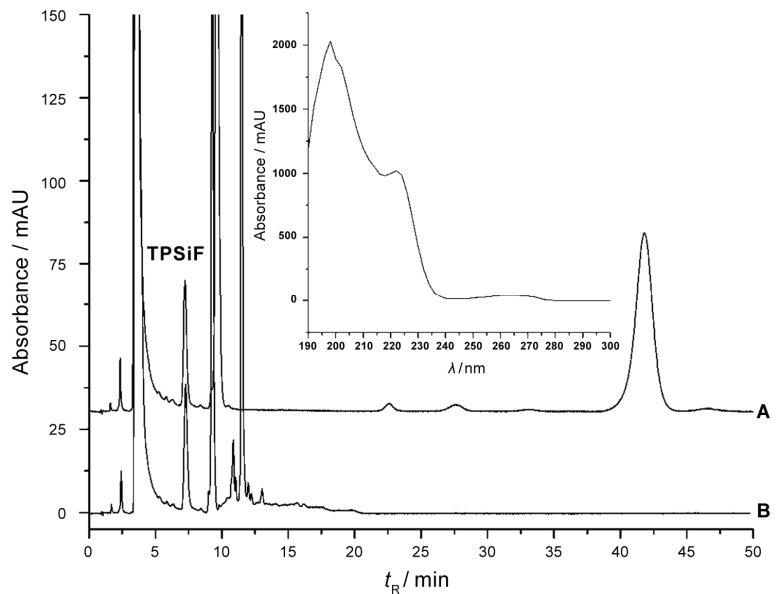

Figure 5. Comparison of chromatograms obtained for TPSiF solution in $n$-hexane with isocratic and gradient elution. Isocratic elution (A) with mobile phase $v(\mathrm{ACN}): v\left(\mathrm{H}_{2} \mathrm{O}\right)=$ $50: 50$ (50\% pump a) and pure ACN (50\% pump b). Gradient elution (B) with mobile phase $v(\mathrm{ACN}): v\left(\mathrm{H}_{2} \mathrm{O}\right)=50: 50(50 \%$ pump a) and pure $\mathrm{ACN}(50 \%$ pump b), after $8 \min 100 \%$ pump b . Injection volume: $15 \mu \mathrm{L}$; UV detection at $222 \mathrm{~nm}$. Inset: TPSiF UV spectrum between 190 and $300 \mathrm{~nm}$.

Modern instrumentation provides a wide range of accessible tools for the development of sensitive and reliable methods of fluoride determination. The fluoride ion-selective electrode (F-ISE) since its invention in 1960-ties and fast introduction to the market is one of the most commonly employed potentiometric electrodes with variety of applications. Its other features were also studied in the past by our group. ${ }^{30,31}$ The analytical literature contains numerous papers showing LOD in the micromolar range, ${ }^{32,33}$ but one can find also LOD of $0.01 \mu \mathrm{mol} \mathrm{L}{ }^{-1}$ reported in FIA system. ${ }^{34}$

Besides the potentiometry with F-ISE, the most important and commonly employed separation technique for fluoride determination is ion chromatography (IC) with conductivity detection with LOD value from 0.14 to $15 \mu \mathrm{mol} \mathrm{L}{ }^{-1}$. The greatest advantage of the method is simultaneous determination of multiple ion species in a single run, however, very short retention time of fluoride ion and therefore proximity of the peak to the void volume signal causes problems with the peak shape and the integration.
The high performance liquid chromatography (HPLC) with reversed-phase (RP) mode columns and the UV detection is a foundation of many analytical methods in the contemporary routine and research laboratories. ${ }^{15}$ It is somewhat surprising that only a very few papers could be found describing the application of RP-HPLC in determination of fluoride. Considering a common use of the HPLC instrumentation nowadays in analytical laboratories, and large variety of available reversed-phase columns, we developed a RP-HPLC method for fluoride determination. The foundation of this method is a reaction originally employed for the determination of fluoride by the gas chromatography. ${ }^{35}$ This method is based on the reaction of hydrogen fluoride with trimethylsilanol to form trimethylfluorosilane. Following this principle we adapted it to the RP-HPLC with UV detection utilizing triphenylsilanol (TPSiOH) in the following reaction $(\mathrm{R}=$ phenyl):

$$
\mathrm{R}_{3} \mathrm{SiOH}+\mathrm{H}^{+}+\mathrm{F}^{-} \rightleftarrows \mathrm{R}_{3} \mathrm{SiF}+\mathrm{H}_{2} \mathrm{O}
$$

The method development included the optimization of derivatization conditions and establishing the HPLC conditions for determination of TOF based on a defluorination procedure with sodium biphenyl reagent $(\mathrm{SBP})^{35}$ used in our earlier FIA measurements. ${ }^{11,12}$

As it was mentioned above, most commonly trace amounts of inorganic fluoride are determined with suppressed IC with conductivity detection. This method in the first step of these studies was examined for determination of fluoride released in reaction with SBP after hydrolysis of reaction mixture. The chromatogram obtained for a standard mixture of anions showed satisfactory separation, however, in the range of retention time corresponding to fluoride, a large tailing peak was recorded. This peak does not allow detection of fluoride in these conditions. It means that the IC can not be employed in this particular case, hence a further attempt was focused on employing the RP-HPLC for detection of fluoride. ${ }^{13}$

The chromatographic parameters that required optimization for the HPLC determination of fluoride, based on its derivatization with TPSiOH were as follows: proper solvent for the extraction of derivatized 


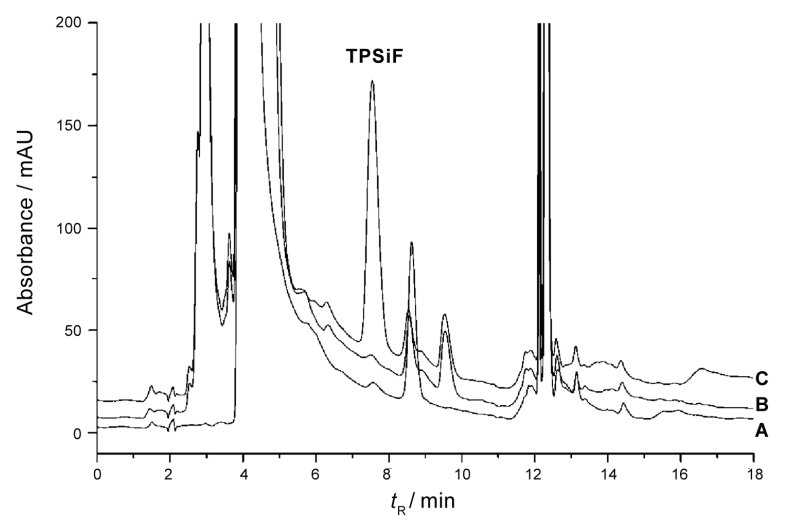

Figure 6. Chromatogram of a blank without SBP (A); chromatogram of a blank after defluorination procedure using SBP and derivatization with TPSiOH (B); chromatogram of a C8PFCA sample processed with defluorination and derivatization procedures $(\mathrm{C})$.

fluoride in the form of triphenylfluorosilane (TPSiF) that was compatible with the chromatographic conditions (column type, mobile phase, gradient conditions) that allow baseline separation of derivatizing reagent (TPSiOH), analyte (TPSiF), and any potential impurities. In addition, the UV detection conditions and the injection volume to provide highest sensitivity and selectivity were also optimized. ${ }^{13}$

The derivatization procedure involves protonation of fluoride present in aqueous sample using a strong mineral acid, reaction of HF with TPSiOH, and extraction of reaction product to hexane layer. The parameters that were optimized for the derivatization procedure included: type and ratio of acid to sample volume, molar ratio of $\mathrm{TPSiOH}$ to fluoride, and reaction time. The derivatization reaction efficiency was calculated as a ratio of the TPSiF concentration obtained using the derivatization procedure, determined from calibration curve prepared using calibration standards of TPSiF in hexane, to the theoretical TPSiF concentration, calculated using known fluoride amount used for the reaction, times 100 percent.

The optimized derivatization procedure was as follows: $2 \mathrm{~mL}$ of aqueous fluoride solution of a given concentration (water in case of blank) was placed in a $10 \mathrm{~mL}$ glass test tube, mixed with $3 \mathrm{~mL}$ of perchloric acid, and TPSiOH (ACN solution, typically added $75-150 \mu \mathrm{L}$ of $0.043 \mathrm{M}$ solution for fluoride in concentration range of $0.25-50 \mu \mathrm{mol} \mathrm{L}^{-1}$ ) in quantity ensuring approximately 100 fold molar excess of $\mathrm{TPSiOH}$ to fluoride. Then, $1 \mathrm{~mL}$ of $n$-hexane was added to the mixture. The mixture was rotated for approximately 60 minutes at room temperature. The phases were separated by a 5 minutes centrifugation at $2000 \mathrm{rpm}$ at room temperature. The hexane layer was aspirated and subjected to the HPLC analysis. The Figure 5 shows a comparison of chromatograms obtained for isocratic (A) and optimized gradient elutions (B).
The limit of detection for fluoride for $\mathrm{S} / \mathrm{N}=3$ for column of $4.6 \mathrm{~mm}$ diameter evaluated as $0.36 \mu \mathrm{mol} \mathrm{L}^{-1}$, while for that one of $2.1 \mathrm{~mm}$ i.d. as $0.09 \mu \mathrm{mol} \mathrm{L}^{-1}$. For the determination of PFOA (C8-PFCA) without preconcentration the limit of detection was evaluated as 2.2 $\mu \mathrm{gl} \mathrm{L} \mathrm{L}^{-1}$.

The developed method for the fluoride determination was evaluated for feasibility of fluoride determination obtained in a procedure of TOF determination. The TOF determination procedure consists of two steps. First, the organic fluorine is transformed into fluoride ions (defluorination step) and then determination of resulting fluoride is conducted. The defluorination step was accomplished using the SBP reagent. PFOA (C8-PFCA) was used here as a model fluoroorganic compound. The amount of C8-PFCA taken for determination provides theoretically $10.4 \mu \mathrm{mol} \mathrm{L}{ }^{-1}$ final fluoride concentration. The blank was processed the same way as the samples with pure acetonitrile instead of C8-PFCA solution. The quality control samples of $10 \mu \mathrm{mol} \mathrm{L} \mathrm{L}^{-1}$ fluoride were derivatized simultaneously with fluoride obtained after defluorination of C8-PFCA. All the results were corrected with a blank value, corresponding to approximately $0.4 \mu \mathrm{mol} \mathrm{L}^{-1}$.

Figure 6 presents comparison of chromatograms of a sample obtained after the combined procedure including defluorination and fluoride derivatization with the blanks. No additional signals overlap with the TPSiF peak, which proves the method usefulness to fluoride determination after defluorination reaction. These initial results showed that the yield of defluorination reaction was $111.2 \pm 5.7 \%$ for ten replicates.

The developed method is an alternative to the TOF determination by the combustion ion chromatography (CIC), ${ }^{9,37,38}$ because there are used only a conventional equipment (HPLC) and for defluorination a commercially available reagent (SBP).

\section{CONCLUSION}

A widely recognized environmental importance of perfluorinated compounds, and their common presence in foods and living organisms require permanent monitoring of their content and ways of transport. Their trace level and a large structural variety are large challenges for modern analytical chemistry. Besides a commonly employed advanced LC/MS methods the common monitoring requires the development of easier accessible methods of determination, isolation and clean-up samples. This role can be played by the presented HPLC method with fluorescence detection or capillary electrophoretic methods with appropriate sample pre-treatment steps, presented in this paper. It seems to be also valuable to develop a relatively simple method for the determination of TOF as one of general parameters of quality of waters and wastes. 


\section{REFERENCES}

1. E. Kissa, Fluorinated Surfactants and Repellents, $2^{\text {nd }}$ Ed., Marcel Dekker Inc., New York 2001.

2. L. Ahrens, S. Felizeter, and R. Ebinghaus, Chemosphere, 76 (2009) 179-184.

3. Directive 2006/122/ECOF the european parliament and of the council.

4. M. A. Andersen, J. L. Butenhoff, S. C. Chang, D. G. Farrar, G. L. Kennedy, C. Lau, G. W. Olsen, J. Seed, and K. B. Wallace, Toxicol. Sci. 102 (2008) 3-14.

5. J. W. Washington, W. M. Henderson, J. J. Ellington, T. M. Jenkins, and J. J. Evans, J. Chromatogr., A 1181 (2008) 21-32

6. T. Ohya, N. Kudo, E. Suzuki, and Y. Kawashima, J. Chromatogr., B: Anal. Technol. Biomed. Life Sci. 720 (1998) 1-7.

7. E. Poboży, E. Król, L. Wójcik, M. Wachowicz, and M. Trojanowicz, Microchim. Acta 172 (2011) 409-417.

8. H. Hori, E. Hayakawa, N. Yamashita, S. Taniyasu, F. Nakata, Y. Kayabaski, Chemosphere 57 (2004) 273-282.

9. L. W. Y. Yeung, Y. Miyake, P. Li, S. Taniyasu, K. Kannan, K. S. Guruge, P. K. S. Lam, and N. Yamashita, Anal. Chim. Acta 6362009 108-114.

10. Y. Miyake, N. Yamashita, M. K. So, P. Rostkowski, S. Taniyasu, P. K. S. Lam, and K. Kannan, J. Chromatogr., A 1154 (2007) 214-221.

11. J. Musijowski, M. Trojanowicz, B. Szostek, J. L. F. Costa Lima, R. Lapa, H. Yamashita, T. Takayanagi, and S. Motomizu, Anal. Chim. Acta 600 (2007) 147-154.

12. T. Takyanagi, H. Yamashita, S. Motomizu, J. Musijowski, and M. Trojanowicz, Talanta 74 (2008) 1224-1230.

13. J. Musijowski, B. Szostek, M. Koc, and M. Trojanowicz, J. Sep. Sci. 33 (2010) 2636-2644.

14. J. W. Martin, K. Kannan, U. Berger, P. De Voogt, J. Field, J. Franklin, J. P. Giesy, T. Harner, D. C. G. Muir, B. Scott, M. Kaiser, U. Järnberg, K. C. Jones, S. A. Mabury, H. Schroeder, M. Simcik, C. Sottani, B. Van Bavel, A. Kärrman, G. Lindström, and S. Van Leeuwen, Environ. Sci. Technol. 38 (2004) 248A-255A

15. P. de Vogt and M. Saez, Trends Anal. Chem. 25 (2006) 326-342.

16. S. P. J. van Leeuwen, C. P. Swart, I. van der Veen, and J. de Boer, J. Chromatogr., A 1216 (2009) 401-409.

17. N. Yamashita, K. Kannan, S. Taniyasu, Y. Horii, T. Okazawa, G. Petrick, and T. Gamo, Environ. Sci. Technol. 38 (2004) $5522-5528$.
18. K. Wille, J. Vanden Busche, H. Noppe, E. De Wulf, P. Van Caeter, C. R. Janssen, H. F. De Brabander, and L. Vanhaecke, J. Chromatogr., A 1217 (2010) 6616-6622.

19. R. Alzaga and J. M. Bayona, J. Chromatogr., A 1042 (2004) $155-162$.

20. V. Dufkova, R. Cabala, D. Maradova, and M. Sticha, $J$ Chromatogr., A 1216 (2009) 8659-8664.

21. C. A. Moody, W. C. Kwan, J. W. Martin, D. C. G. Muir, and S. A. Mabury, Anal. Chem. 73 (2001) 2200-2206.

22. M. S. Maclachlan, K. E. Holmstrom, M. Reth, and U. Berger, Environ. Sci. Technol. 41 (2007) 7260-7265.

23. J. Peris Vicente, J. V. Gimeno Adelantado, M. T. Domenech Carbo, R. Mateo Castro, and F. Bosch Reig, J. Chromatogr., A 1076 (2005) 44-50.

24. L. Arens, M. Plassmann, Z. Xie, and R. Ebinghaus, Front. Environ. Sci. Engin. China 3 (2009) 152-170.

25. S. P. J. van Leeuwen and J. De Boer, J. Chromatogr., A 1153 (2007) 172-185.

26. L. Wójcik, B. Szostek, W. Maruszak, and M. Trojanowicz, Electrophoresis 26 (2005) 1080-1088.

27. M. Masar, L. Wójcik, D. Kaniansky, and M. Trojanowicz, J. Sep. Sci. 28 (2005) 1271-1277.

28. P. Doble, M. Macka, and P. R. Haddad, Trends Anal. Chem. 19 (2000) 10-17.

29. M. Trojanowicz, J. Musijowski, M. Koc, and M. A. Donten, Anal. Methods 3 (2011) 1039-1045.

30. M. Trojanowicz, Talanta 26 (1979) 985-986.

31. M. Trojanowicz and W. Frenzel, Fresenius Z. Anal. Chem. 328 (1987) 653-656.

32. M. Ponikvar, V. Stibilj, and B. Zemva, Food Chem. 103 (2007) 369-374.

33. T.-H. Shyu, J.-H. Chen, and Y.-H. Lee, J. Food Drug Anal. 17 (2009) 22-27.

34. H. Hara and C.-C. Huang, Anal. Chim. Acta 338 (1997) 141-147.

35. J. A. Fresen, F. H. Cox, and M. J. Witter, Pharm. Weekblad 103 (1968) 909-914.

36. K. Mackenzie, F.-D. Kopinke, and M. Remmler, Chemosphere 33 (1996) 1495-1513.

37. L. W. Y. Yeung, Y. Miyake, S. Taniyasu, Y. Wang, H. Yu, M. K. So, G. Jiang, Y. Wu, J. Li, J. P. Giesy, N. Yamashita, and P. K. S. Lam, Environ. Sci. Technol. 42 (2008) 8140-8145.

38. L. W. Y. Yeung, Y. Miyake, Y. Wang, S. Taniyasu, N. Yamashita, and P. K .S. Lam, Environ. Pollut. 157 (2009) 17-23. 